\title{
Energy Decay of Vortices in Viscous Fluids: an Applied Mathematics View
}

\author{
Jan Nordström and Björn Lönn
}

\section{Linköping University Post Print}

N.B.: When citing this work, cite the original article.

Original Publication:

Jan Nordström and Björn Lönn, Energy Decay of Vortices in Viscous Fluids: an Applied Mathematics View, 2012, Journal of Fluid Mechanics.

http://dx.doi.org/10.1017/jfm.2012.351

Copyright: Cambridge University Press (CUP)

http://www.cambridge.org/uk/

Postprint available at: Linköping University Electronic Press

http://urn.kb.se/resolve?urn=urn:nbn:se:liu:diva-80799 


\title{
Energy Decay of Vortices in Viscous Fluids: an Applied Mathematics View
}

\author{
JA N NORDSTRÖ M $\mathbf{M}^{1} \dagger$ AND BJ ÖRN L Ö N N ${ }^{2}$ \\ ${ }^{1}$ Department of Mathematics, Linköping University, SE-581 83 Linköping, Sweden \\ ${ }^{2}$ Department of Information Technology, Uppsala University, SE-75105 Uppsala, Sweden \\ (Received ?; revised ?; accepted ?. - To be entered by editorial office)
}

The energy decay of vortices in viscous fluids governed by the compressible NavierStokes equations is investigated. It is shown that the main reason for the slow decay is that zero eigenvalues exist in the matrix related to the dissipative terms. The theoretical analysis is purely mathematical and based on the energy method. To check the validity of the theoretical result in practice, numerical solutions to the Navier-Stokes equations are computed using a stable high order finite difference method. The numerical computations corroborate the theoretical conclusion.

Key words:

\section{Introduction}

Vortices are common flow features in aeronautical applications. The topic of this paper, the slow energy decay, causes problems when wingtip vortices remain on the runway for a long time, see Proctor (1998), Kantha (2010), Gerz et al. (2002), Breitsamter (2011), Spalart (1998). Vortices are also important when explaining several flow phenomena. The lift generated by wings is investigated in Chen et al. (2010), Rossow (1999), Yen (2011). It is concluded that effects from leading edge vortices can be used to increase lift.

Vortices are frequently present in nature and also influence the environment. The presence of von Karman vortices in the trailing wind of islands can have effects on the local climate as well as the sea life, see Beggs et al. (2004), Ponta (2010), Holzapfel et al. (2003). Their effect on fish, bird and insect behavior is studied in Wu (2011), Kim \& Kim (2011), Liu et al. (2009). Vortices are also needed when modeling the general circulation of the ocean as seen in Holland (1978), Zhang et al. (2011), Winter \& Bourqui (2011), Koszalka et al. (2011). Other effects include emissions from air traffic Gerz \& Holzapfel (1999) and influence on the efficiency of turbine parks Magnusson \& Smedman (1999).

It is well known that vortices do not decay in inviscid flows governed by the Euler equations. It is quite straightforward to construct exact solutions that advect with a constant background velocity, see Anderson (1991), McCormack \& Crane (1973), Erlebacher et al. (1997), Davoudzadeh et al. (1995). Less well known is why this slow energy decay seem to persist also in viscous flows governed by the Navier-Stokes equations. This phenomena has been thoroughly investigated and many theories of what phenomena are important and why the decay is slow exist, see for example Sarpkaya (1998), Matthaeus et al. (1991), Gustavsson (1991), Greene (1986), Balmforth et al. (2001), Greene (1986), Zhou et al. (1999), Wallin \& Girimaji (2000), Pullin \& Saffman (1998), Winckelmans et al. (2006),

$\dagger$ Email address for correspondence: jan.nordstrom@liu.se 
Kundu \& Cohen (2001). Related but more fundamental theoretical questions like stability and existence of solutions for long times were studied in Hoff \& Zumbrum (1995), Hagstrom \& Lorenz (1995) and Hagstrom \& Lorenz (2002).

Most of the previous investigations and theories are based on observations of measured or computed data and a subsequent analysis. We will take a different approach and rely on mathematics only. This will eliminate potential error sources when producing data, make the analysis transparent to the reader and hence enable the reader to follow and check the whole procedure. Only afterwards, when the main theoretical conclusion has been reached, and the suggested decay scenario has been formulated will the implications of the theory be tested using results from numerical calculations.

We begin by observing that vortices exist for all ranges of Reynolds numbers (with the exception of Stokes flow) including inviscid flows. This indicate that the size of the viscous terms is not of fundamental importance. Hence, it is relevant to analyse a simple laminar flow case, without considering complicated additional phenomena such as turbulence. We will analyse the energy decay of small scale disturbances by applying the standard tool in applied mathematics/numerical analysis, namely the energy-method (see Kreiss \& Lorenz (1989) and Gustafsson et al. (1995) and the numerous references therein). In particular we will investigate the viscous terms and their role in the energy dissipation.

\section{Analysis}

Consider the flow situation when small disturbances are imposed on a constant background state. This could be a model of the situation right after the passing of an aircraft in free space. This problem setup also naturally allows for the linearized analysis below where we neglect quadratic terms and investigate the decay of small scale disturbances.

The dependent variables are the density $\tilde{\rho}$, the velocities in each direction $\tilde{u}, \tilde{v}, \tilde{w}$ and the temperature $\tilde{T}$. The tilde sign signifies the presence of dimensions. We write the equations in non-dimensional form using the free stream density $\tilde{\rho}_{\infty}$, the free stream velocity $\tilde{U}_{\infty}$ and the free stream temperature $\tilde{T}_{\infty}$. The shear and second viscosity coefficients $\tilde{\mu}, \tilde{\lambda}$ as well as the coefficient of heat conduction $\tilde{\kappa}$ are non-dimensionalized with the free stream viscosity $\tilde{\mu}_{\infty}$. The pressure in non-dimensional form becomes: $p=\tilde{p} /\left(\tilde{\rho}_{\infty} \tilde{U}_{\infty}^{2}\right)=\rho T /\left(\gamma M_{\infty}^{2}\right)$. Also used are

$$
M_{\infty}^{2}=\frac{\tilde{U}_{\infty}^{2}}{\gamma \tilde{R} \tilde{T}_{\infty}}, \quad \operatorname{Pr}=\frac{\tilde{\mu}_{\infty} C_{p}}{\tilde{\kappa}_{\infty}}, \quad \operatorname{Re}=\frac{\tilde{\rho}_{\infty} \tilde{U}_{\infty} \tilde{L}}{\tilde{\mu}_{\infty}}, \quad \gamma=\frac{C_{p}}{C_{v}}, \quad \varphi=\frac{\gamma \kappa}{\operatorname{Pr}}
$$

where $\tilde{L}$ is a length scale and $M, \operatorname{Pr}, \operatorname{Re}=1 / \epsilon$ and $\gamma$ are the Mach-, Prandtl-, Reynolds number and ratio of specific heats respectively. The time scale is $\tilde{L} / \tilde{U}_{\infty}$.

\subsection{The energy method}

The linearized dimensionless compressible Navier-Stokes equations can formally be written

$$
\begin{gathered}
\frac{\partial V}{\partial t}+\bar{A} \frac{\partial V}{\partial x}+\bar{B} \frac{\partial V}{\partial y}+\bar{C} \frac{\partial V}{\partial z}=\frac{\epsilon}{\bar{\rho}}\left[\left(\bar{D}_{11} \frac{\partial V}{\partial x}+\bar{D}_{12} \frac{\partial V}{\partial y}+\bar{D}_{13} \frac{\partial V}{\partial z}\right)_{x}+\right. \\
\left.+\left(\bar{D}_{21} \frac{\partial V}{\partial x}+\bar{D}_{22} \frac{\partial V}{\partial y}+\bar{D}_{23} \frac{\partial V}{\partial z}\right)_{y}+\left(\bar{D}_{31} \frac{\partial V}{\partial x}+\bar{D}_{32} \frac{\partial V}{\partial y}+\bar{D}_{33} \frac{\partial V}{\partial z}\right)_{z}\right] .
\end{gathered}
$$

In (2.2), $V=(\rho, u, v, w, T)^{T}$ is the disturbance and the constant state around which we linearize is denoted by an overbar. The matrices related to the hyperbolic terms in (2.2) must be symmetric for the energy method to be applicable Nordström \& Svärd (2005). 
In Abarbanel \& Gottlieb (1981) it is shown that the symmetrization can be done using a single matrix $S$. We choose the symmetrizer

$$
S^{-1}=\operatorname{diag}\left[\frac{\bar{c}^{2}}{\sqrt{\gamma}}, \bar{\rho} \bar{c}, \bar{\rho} \bar{c}, \bar{\rho} \bar{c}, \frac{\bar{\rho}}{\sqrt{\gamma(\gamma-1) M_{\infty}^{4}}}\right],
$$

where $\bar{c}$ is the speed of sound at reference state.

Remark: Abarbanel \& Gottlieb (1981) showed that the three-dimensional compressible Navier-Stokes equations with 12 matrices involved (see (2.2)), can be symmetrized by a single matrix (symmetrizer). This remarkable fact was complemented by the observation that there are at least two different symmetrizers, based on either the hyperbolic or the parabolic terms. The symmetrizer (2.3) is related to the parabolic terms.

The similarity transform is applied by multiplying (2.2) from the left with $S^{-1}$. After applying the symmetrizer we obtain

$$
\begin{aligned}
& \tilde{A}=\left(\begin{array}{ccccc}
\bar{u} & \bar{c} / \sqrt{\gamma} & 0 & 0 & 0 \\
\bar{c} / \sqrt{\gamma} & \bar{u} & 0 & 0 & \bar{c} \sqrt{\frac{\gamma-1}{\gamma}} \\
0 & 0 & \bar{u} & 0 & 0 \\
0 & 0 & 0 & \bar{u} & 0 \\
0 & \bar{c} \sqrt{\frac{\gamma-1}{\gamma}} & 0 & 0 & \bar{u}
\end{array}\right) \quad \bar{D}_{11}=\left(\begin{array}{ccccc}
0 & 0 & 0 & 0 & 0 \\
0 & 2 \bar{\mu}+\bar{\lambda} & 0 & 0 & 0 \\
0 & 0 & \bar{\mu} & 0 & 0 \\
0 & 0 & 0 & \bar{\mu} & 0 \\
0 & 0 & 0 & 0 & \bar{\varphi}
\end{array}\right) \\
& \tilde{B}=\left(\begin{array}{ccccc}
\bar{v} & 0 & \bar{c} / \sqrt{\gamma} & 0 & 0 \\
0 & \bar{v} & 0 & 0 & 0 \\
\bar{c} / \sqrt{\gamma} & 0 & \bar{v} & 0 & \bar{c} \sqrt{\frac{\gamma-1}{\gamma}} \\
0 & 0 & 0 & \bar{v} & 0 \\
0 & 0 & \bar{c} \sqrt{\frac{\gamma-1}{\gamma}} & 0 & \bar{v}
\end{array}\right) \quad \bar{D}_{22}=\left(\begin{array}{ccccc}
0 & 0 & 0 & 0 & 0 \\
0 & \bar{\mu} & 0 & 0 & 0 \\
0 & 0 & 2 \bar{\mu}+\bar{\lambda} & 0 & 0 \\
0 & 0 & 0 & \bar{\mu} & 0 \\
0 & 0 & 0 & 0 & \bar{\varphi}
\end{array}\right) \\
& \tilde{C}=\left(\begin{array}{ccccc}
\bar{w} & 0 & 0 & \bar{c} / \sqrt{\gamma} & 0 \\
0 & \bar{w} & 0 & 0 & 0 \\
0 & 0 & \bar{w} & 0 & 0 \\
\bar{c} / \sqrt{\gamma} & 0 & 0 & \bar{w} & \bar{c} \sqrt{\frac{\gamma-1}{\gamma}} \\
0 & 0 & 0 & \bar{c} \sqrt{\frac{\gamma-1}{\gamma}} & \bar{w}
\end{array}\right) \quad \bar{D}_{33}=\left(\begin{array}{ccccc}
0 & 0 & 0 & 0 & 0 \\
0 & \bar{\mu} & 0 & 0 & 0 \\
0 & 0 & \bar{\mu} & 0 & 0 \\
0 & 0 & 0 & 2 \bar{\mu}+\bar{\lambda} & 0 \\
0 & 0 & 0 & 0 & \bar{\varphi}
\end{array}\right) \\
& \bar{D}_{12}=\bar{D}_{21}^{T}=\left(\begin{array}{ccccc}
0 & 0 & 0 & 0 & 0 \\
0 & 0 & \bar{\lambda} & 0 & 0 \\
0 & \bar{\mu} & 0 & 0 & 0 \\
0 & 0 & 0 & 0 & 0 \\
0 & 0 & 0 & 0 & 0
\end{array}\right) \quad \bar{D}_{13}=\bar{D}_{31}^{T}=\left(\begin{array}{ccccc}
0 & 0 & 0 & 0 & 0 \\
0 & 0 & 0 & \bar{\lambda} & 0 \\
0 & 0 & 0 & 0 & 0 \\
0 & \bar{\mu} & 0 & 0 & 0 \\
0 & 0 & 0 & 0 & 0
\end{array}\right) \\
& \bar{D}_{23}=\bar{D}_{32}^{T}=\left(\begin{array}{ccccc}
0 & 0 & 0 & 0 & 0 \\
0 & 0 & 0 & 0 & 0 \\
0 & 0 & 0 & \bar{\lambda} & 0 \\
0 & 0 & \bar{\mu} & 0 & 0 \\
0 & 0 & 0 & 0 & 0
\end{array}\right) \\
& \tilde{V}=\left(\begin{array}{c}
\frac{\bar{c}^{2}}{\sqrt{\gamma}} \rho \\
\bar{\rho} \bar{c} u \\
\bar{\rho} \bar{c} v \\
\bar{\rho} \bar{c} w \\
\frac{\bar{\rho}}{\sqrt{\gamma(\gamma-1) M_{\infty}^{4}}} T
\end{array}\right)
\end{aligned}
$$

where $\tilde{V}^{T}=\left(S^{-1} V\right)^{T}, \tilde{A}=S^{-1} \bar{A} S, \tilde{B}=S^{-1} \bar{B} S, \tilde{C}=S^{-1} \bar{C} S$ and the $\bar{D}_{i j}$ 's are 
unchanged. The matrices above are all given in Abarbanel \& Gottlieb (1981) but we list them here for completeness.

The energy method is applied by multiplying the symmetrized dimensionless NavierStokes equations with $\tilde{V}^{T}$ and integrating over the domain $\Omega$. Let the energy norm be defined as $\|\tilde{V}\|^{2}=\int_{\Omega} \tilde{V}^{T} \tilde{V} d x d y d z$. By using Gauss' theorem and integration by parts, we obtain

$$
\|\tilde{V}\|_{t}^{2}+B T=-2\left(\frac{\epsilon}{\bar{\rho}}\right) \int_{\Omega}\left(\begin{array}{c}
\tilde{V}_{x} \\
\tilde{V}_{y} \\
\tilde{V}_{z}
\end{array}\right)^{T}\left(\begin{array}{ccc}
\bar{D}_{11} & \bar{D}_{12} & \bar{D}_{13} \\
\bar{D}_{21} & \bar{D}_{22} & \bar{D}_{23} \\
\bar{D}_{31} & \bar{D}_{32} & \bar{D}_{33}
\end{array}\right)\left(\begin{array}{c}
\tilde{V}_{x} \\
\tilde{V}_{y} \\
\tilde{V}_{z}
\end{array}\right) d x d y d z
$$

where

$$
B T=\oint_{\partial \Omega}\left(\begin{array}{c}
\tilde{V}^{T}\left[\tilde{A} \tilde{V}-2\left(\frac{\epsilon}{\bar{\rho}}\right)\left(\bar{D}_{11} \tilde{V}_{x}+\bar{D}_{12} \tilde{V}_{y}+\bar{D}_{13} \tilde{V}_{z}\right)\right] \\
\tilde{V}^{T}\left[\tilde{B} \tilde{V}-2\left(\frac{\epsilon}{\bar{\rho}}\right)\left(\bar{D}_{21} \tilde{V}_{x}+\bar{D}_{22} \tilde{V}_{y}+\bar{D}_{23} \tilde{V}_{z}\right)\right] \\
\tilde{V}^{T}\left[\tilde{C} \tilde{V}-2\left(\frac{\epsilon}{\bar{\rho}}\right)\left(\bar{D}_{31} \tilde{V}_{x}+\bar{D}_{32} \tilde{V}_{y}+\bar{D}_{33} \tilde{V}_{z}\right)\right]
\end{array}\right) \cdot \hat{n} d s
$$

In (2.10), $d s=\sqrt{d x^{2}+d y^{2}+d z^{2}}$ and $\hat{n}=\left(n_{1}, n_{2}, n_{3}\right)^{T}$ is the outward pointing unit normal on the surface $\partial \Omega$.

In this paper we ignore the effect of far-field boundaries and consider the Cauchy problem only. In particular, we focus on the volume term on the right-hand-side of (2.9) and neglect the boundary term BT. Let the block matrix consisting of the $\bar{D}_{i j}$ matrices be denoted by $\bar{D}$. If $\bar{D}$ is positive semi-definite, (2.9) show that the energy decays.

Remark: Normally the relations (2.9) and (2.10) are used in order to determine boundary conditions that lead to well-posedness, see for example Gustafsson \& Sundström (1978), Nordström (1995) and Nordström \& Svärd (2005). In that case the focus is on the term BT.

Remark: If $\bar{D}$ would not be positive semi-definite, then (2.9) would show that the Cauchy problem for the linearized Navier-Stokes equation is not well posed.

The first, sixth and 11th rows as well as the first, sixth and 11th columns of the $15 \times 15$ matrix $\bar{D}$ have only zero elements, thus the matrix can be reduced to a $12 \times 12$ matrix. We refer to this matrix as $\hat{D}$.

$$
\hat{D}=\left(\begin{array}{cccccccccccc}
2 \mu+\lambda & 0 & 0 & 0 & 0 & \lambda & 0 & 0 & 0 & 0 & \lambda & 0 \\
0 & \mu & 0 & 0 & \mu & 0 & 0 & 0 & 0 & 0 & 0 & 0 \\
0 & 0 & \mu & 0 & 0 & 0 & 0 & 0 & \mu & 0 & 0 & 0 \\
0 & 0 & 0 & \varphi & 0 & 0 & 0 & 0 & 0 & 0 & 0 & 0 \\
0 & \mu & 0 & 0 & \mu & 0 & 0 & 0 & 0 & 0 & 0 & 0 \\
\lambda & 0 & 0 & 0 & 0 & 2 \mu+\lambda & 0 & 0 & 0 & 0 & \lambda & 0 \\
0 & 0 & 0 & 0 & 0 & 0 & \mu & 0 & 0 & \mu & 0 & 0 \\
0 & 0 & 0 & 0 & 0 & 0 & 0 & \varphi & 0 & 0 & 0 & 0 \\
0 & 0 & \mu & 0 & 0 & 0 & 0 & 0 & \mu & 0 & 0 & 0 \\
0 & 0 & 0 & 0 & 0 & 0 & \mu & 0 & 0 & \mu & 0 & 0 \\
\lambda & 0 & 0 & 0 & 0 & \lambda & 0 & 0 & 0 & 0 & 2 \mu+\lambda & 0 \\
0 & 0 & 0 & 0 & 0 & 0 & 0 & 0 & 0 & 0 & 0 & \varphi
\end{array}\right)
$$

The eigenvalues of $\hat{D}$ are obtained by solving $\operatorname{det}(\hat{D}-s I)=0$ for $s$ which yield $s=$ $\varphi, \varphi, \varphi, 2 \mu, 2 \mu, 2 \mu, 0,0,0,2 \mu, 2 \mu, 2 \mu+3 \lambda$. The second law of thermodynamics, see White (1974), imply that $2 \mu+3 \lambda \geqslant 0$ and hence all the eigenvalues are positive semi-definite as required for a well posed problem.

To see how the velocity gradients and the eigenvalues are related, the eigenvectors 
must be calculated. The orthonormal matrix with the eigenvectors as columns is

$$
Q=\left(\begin{array}{cccccccccccc}
0 & 0 & 0 & 0 & 0 & 0 & 0 & 0 & 0 & \sqrt{\frac{1}{6}} & \frac{1}{\sqrt{2}} & \frac{1}{\sqrt{3}} \\
0 & 0 & 0 & \frac{1}{\sqrt{2}} & 0 & 0 & \frac{1}{\sqrt{2}} & 0 & 0 & 0 & 0 & 0 \\
0 & 0 & 0 & 0 & \frac{1}{\sqrt{2}} & 0 & 0 & \frac{1}{\sqrt{2}} & 0 & 0 & 0 & 0 \\
1 & 0 & 0 & 0 & 0 & 0 & 0 & 0 & 0 & 0 & 0 & 0 \\
0 & 0 & 0 & \frac{1}{\sqrt{2}} & 0 & 0 & \frac{-1}{\sqrt{2}} & 0 & 0 & 0 & 0 & 0 \\
0 & 0 & 0 & 0 & 0 & 0 & 0 & 0 & 0 & -\sqrt{\frac{4}{6}} & 0 & \frac{1}{\sqrt{3}} \\
0 & 0 & 0 & 0 & 0 & \frac{1}{\sqrt{2}} & 0 & 0 & \frac{1}{\sqrt{2}} & 0 & 0 & 0 \\
0 & 1 & 0 & 0 & 0 & 0 & 0 & 0 & 0 & 0 & 0 & 0 \\
0 & 0 & 0 & 0 & \frac{1}{\sqrt{2}} & 0 & 0 & \frac{-1}{\sqrt{2}} & 0 & 0 & 0 & 0 \\
0 & 0 & 0 & 0 & 0 & \frac{1}{\sqrt{2}} & 0 & 0 & \frac{-1}{\sqrt{2}} & 0 & 0 & 0 \\
0 & 0 & 0 & 0 & 0 & 0 & 0 & 0 & 0 & \sqrt{\frac{1}{6}} & -\frac{1}{\sqrt{2}} & \frac{1}{\sqrt{3}} \\
0 & 0 & 1 & 0 & 0 & 0 & 0 & 0 & 0 & 0 & 0 & 0
\end{array}\right) .
$$

We obtain $\hat{D}=Q \Lambda Q^{T}$ where $\Lambda=\operatorname{diag}(\varphi, \varphi, \varphi, 2 \mu, 2 \mu, 2 \mu, 0,0,0,2 \mu, 2 \mu, 2 \mu+3 \lambda)$. By inserting $\hat{D}$ (or the identical $\bar{D}$ ) into $(2.9)$ we obtain the final form of the energy decay rate

$$
\|\tilde{V}\|_{t}^{2}=-2\left(\frac{\epsilon}{\bar{\rho}}\right) \int_{D}\left[Q^{T}\left(\begin{array}{c}
\tilde{V}_{x} \\
\tilde{V}_{y} \\
\tilde{V}_{z}
\end{array}\right)\right]^{T} \Lambda\left[Q^{T}\left(\begin{array}{c}
\tilde{V}_{x} \\
\tilde{V}_{y} \\
\tilde{V}_{z}
\end{array}\right)\right] d x d y d z
$$

where

$$
Q^{T}\left(\begin{array}{c}
\tilde{V}_{x} \\
\tilde{V}_{y} \\
\tilde{V}_{z}
\end{array}\right)=\left(\begin{array}{c}
\frac{\bar{\rho}}{\sqrt{\gamma(\gamma-1) M_{\infty}^{4}}} T_{x} \\
\frac{\bar{\rho}}{\sqrt{\gamma(\gamma-1) M_{\infty}^{4}}} T_{y} \\
\frac{\bar{\rho}}{\sqrt{\gamma(\gamma-1) M_{\infty}^{4}}} T_{z} \\
\frac{1}{\sqrt{2}} \bar{\rho} \bar{c}\left[v_{x}+u_{y}\right] \\
\frac{1}{\sqrt{2}} \bar{\rho} \bar{c}\left[w_{x}+u_{z}\right] \\
\frac{1}{\sqrt{2}} \bar{\rho} \bar{c}\left[w_{y}+v_{z}\right] \\
\frac{1}{\sqrt{2}} \bar{\rho} \bar{c}\left[v_{x}-u_{y}\right] \\
\frac{1}{\sqrt{2}} \bar{\rho} \bar{c}\left[w_{x}-u_{z}\right] \\
\frac{1}{\sqrt{2}} \bar{\rho} \bar{c}\left[w_{y}-v_{z}\right] \\
\left.\sqrt{\frac{2}{3}} \bar{\rho} \bar{c} \frac{1}{2} u_{x}-v_{y}+\frac{1}{2} w_{z}\right] \\
\frac{1}{\sqrt{2}} \bar{\rho} \bar{c}\left[u_{x}-w_{z}\right] \\
\frac{1}{\sqrt{3}} \overline{\bar{\rho}} \bar{c}\left[u_{x}+v_{y}+w_{z}\right]
\end{array}\right) .
$$

\subsection{Analysis of the energy decay rate}

The relation (2.13) and (2.14) show the relation between eigenvalues and gradients. The temperature gradients are multiplied by the eigenvalue $\varphi$. The following three components consist of terms related to the rotation of the velocity field in the xy-, xz- and yz planes and are each multiplied by $2 \mu$. The most interesting fact is that the vorticity components in each plane are multiplied by a zero eigenvalue. The last term is the divergence and it is multiplied by the positive eigenvalue $2 \mu+3 \lambda$. The two remaining terms consist of components related to the divergence $u_{x}+v_{y}+w_{z}$ and they are multiplied by $2 \mu$.

Based on (2.13) and (2.14) we can now draw the two central conclusions of this paper: 
- The vorticity terms are multiplied by zero eigenvalues.

- The decay rate is proportional to the gradients squared.

These conclusions suggest that vortices will decay slowly because the main part of the energy is concentrated in the vorticity components. They also indicate that flow structures with gradients that are not in vorticity form will decay fast.

Remark: The velocity gradient can be decomposed into symmetric and antisymmetric parts (strain-rate and vorticity tensors). The eigenvalues associated with the vorticity tensor are zero while eigenvalues associated with the strain-rate tensor are non-zero. The only flow condition that has zero strain rate and non-zero vorticity is solid body rotation, which, according to the analysis, is the only flow that will be undamped. All other kind of vortical flows will be damped since it will contain some amount of strain rate.

Remark: The analysis above show that if all the energy reside in the vorticity components, there is no energy decay. However, the energy can be transferred to other modes and lead to decay. If the transfer is slow, the energy decay will be small. On the other hand it is also possible to have an energy decay that halts due to the transfer of energy from non-vorticity modes into vorticity modes. It is not possible (although indications exist, see Hoff \& Zumbrum (1995)) to analytically determine whether such a transfer exist and how fast it is, but we will investigate this below by performing numerical calculations.

\section{Numerical calculations}

The computations are done using a well tested and provable stable high order finite difference scheme for the compressible Navier-Stokes equations. It uses summation-byparts operators and imposes boundary and interface conditions weakly as described in Carpenter et al. (1999), Nordström \& Carpenter (1999), Nordström \& Carpenter (2001), Mattsson et al. (2004), Svärd et al. (2007), Svärd \& Nordström (2008) and Nordström et al. (2009). The code runs with 2,3,4 and 5th order global accuracy. The 3rd order accurate scheme with far field boundaries sufficiently far from the actual flow structure was used.

\subsection{The grids and order of accuracy}

The grid used for calculations in two dimensions is each divided into 16 blocks. All blocks have an equal number of grid points. The grid used, for the displayed computations, is a square with side length 20 and 257 points each in both the $\mathrm{x}$ and $\mathrm{y}$ directions. Hence the mesh has $\Delta x=\Delta y=0.078$. For calculations in three dimensions the grid is a cube with side length 20 and it has 129 points in each direction giving $\Delta x=\Delta y=\Delta z=0.155$. The 3rd order accurate scheme was evaluated in two dimensions using the 33-65-129, 65129-257 and 129-257-513 point grids. The practical order of accuracy obtained was 1.71 , 2.18 and 2.40 respectively. The error level in $L_{2}$ norm for the displayed 2D computations is estimated to $3 \times 10^{-7}$.

\subsection{Initial conditions in two dimensions}

To verify the analytical results, long time simulations with three different initial conditions have been investigated. The Reynolds numbers considered are $R e=10,100$ and $R e=1000$. We stress here that the size of the Reynolds number is of no fundamental importance in this investigation. The zero eigenvalues are present for all Reynolds numbers. We have compared the energy decay of a vortex, with the energy decay of white noise, and a specially constructed divergence free flow case. As initial values for all these two dimensional calculations we used: the Mach number $\mathrm{M}=0.005$, the radius $\mathrm{r}=1$, the density and temperature are $\rho_{\infty}=1, T_{\infty}=1$. 


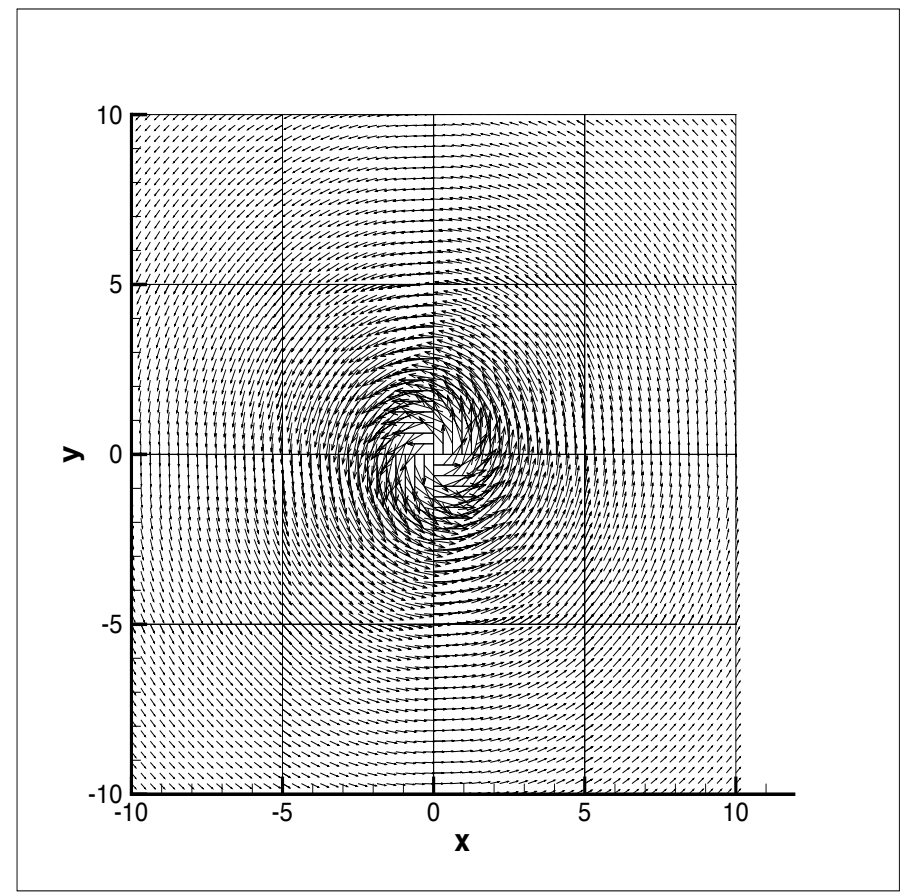

Figure 1: The initial field for the vortex. The velocities decrease fast outside $r=1$.

The vortex is a so called wake vortex also used in Winckelmans et al. (2006). The initial velocity field is shown in Figure 1. It is defined by

$$
u_{i j}=-\frac{\varepsilon \cdot y_{i j}}{x_{i j}^{2}+y_{i j}^{2}+r_{c}^{2}} \quad v_{i j}=\frac{\varepsilon \cdot x_{i j}}{x_{i j}^{2}+y_{i j}^{2}+r_{c}^{2}}
$$

where $x_{i j}, y_{i j}$ are the distances from the center of the vortex in each direction, $r_{c}$ is the distance from the center to the strongest velocity of the vortex and $\varepsilon=2 * r_{c} * M$. For completeness we also give the velocity components in cylindrical coordinates as

$$
U_{\theta}=\frac{\varepsilon r}{r^{2}+r_{c}^{2}} \quad U_{r}=0
$$

where $r=\sqrt{x^{2}+y^{2}}$ is the radius and $U_{\theta}, U_{r}$ are the tangential and radial velocities respectively.

The initial velocity field of the white noise is shown in Figure 2. It is defined by

$$
u_{i j}=\frac{\varepsilon \cdot \text { random }}{x_{i j}^{2}+y_{i j}^{2}+r_{c}^{2}} \quad v_{i j}=\frac{\varepsilon \cdot \text { random }}{x_{i j}^{2}+y_{i j}^{2}+r_{c}^{2}} \quad \text { rand } \in[-1,1]
$$

where $\epsilon$ is the same as in (3.1). The velocities are randomized using random numbers from the Fortran 90 random subroutine and damped to zero using the same function as the vortex condition. The velocities in cylindrical coordinates are

$$
U_{\theta}(r, \theta)=\frac{\varepsilon \text { random }}{r^{2}+r_{c}^{2}}, \quad U_{r}(r, \theta)=\frac{\varepsilon \text { random }}{r^{2}+r_{c}^{2}}
$$

with the same definitions as in (3.2).

In all the calculations in this paper, the Mach number is small, and the initial condition should (almost) satisfy the zero divergence relation in incompressible flow. However, the 


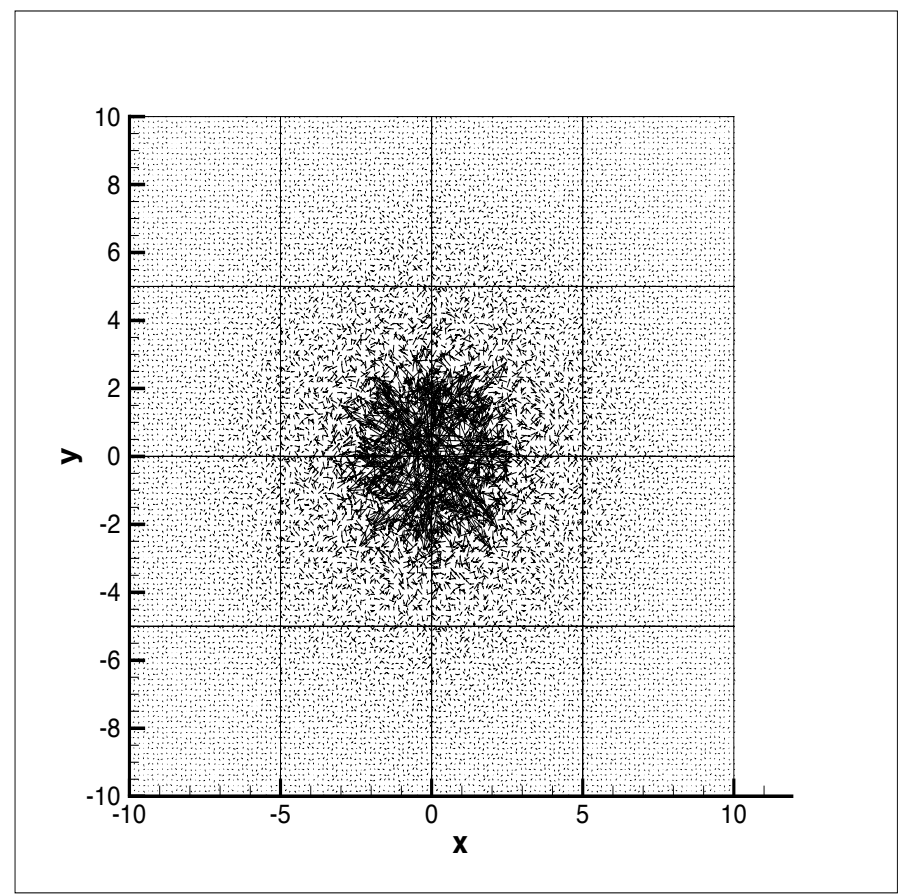

Figure 2: The initial field for the white noise. The velocities decrease fast outside $r=1$.

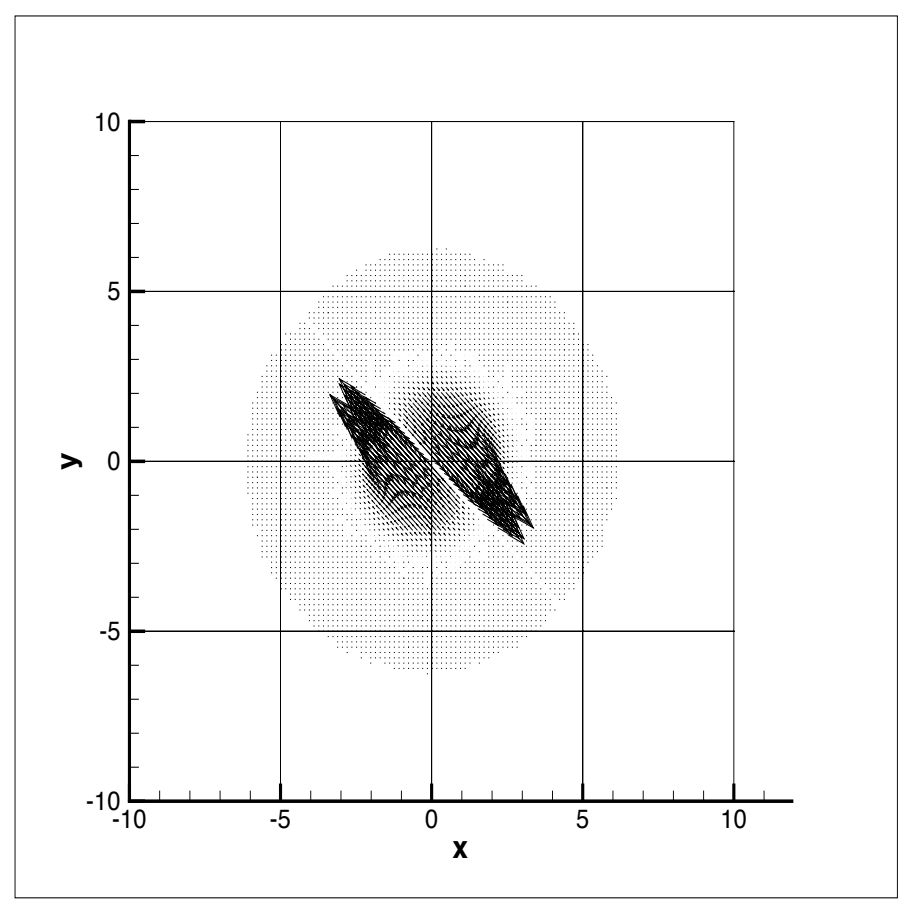

Figure 3: The initial field for the divergence free case. The velocities decrease fast outside $\mathrm{r}=1$. 


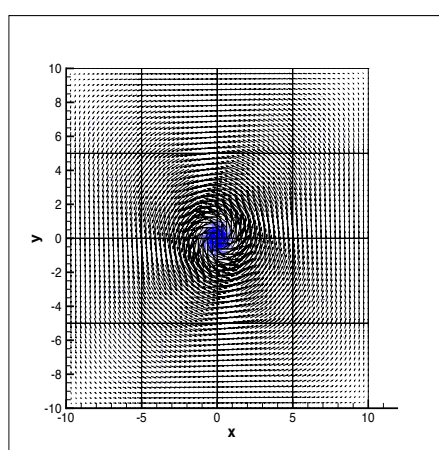

(a) $\mathrm{t}=50$

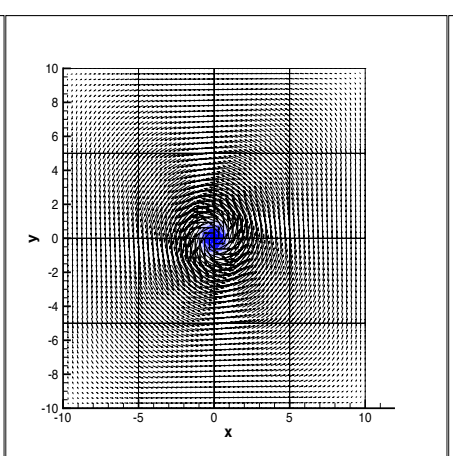

(b) $t=1000$

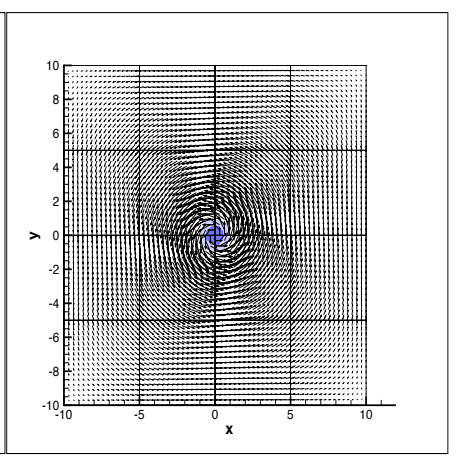

(c) $\mathrm{t}=4000$

Figure 4: Time evolution of the vortex for $R e=1000$. The radius is displayed by the low density core indicated with dark blue. As time passes, the velocities slowly weaken.

white noise do not have zero divergence. In order to check if that has any impact on the results, we included a special zero divergence initial condition shown in Figure 3 of the following form

$$
u_{i j}=\varepsilon \frac{x_{i j}+y_{i j}}{\exp \left[\left(x_{i j}+y_{i j}\right)^{2}+r_{c}^{2}\right]} \quad v_{i j}=-\varepsilon \frac{x_{i j}+y_{i j}}{\exp \left[\left(x_{i j}+y_{i j}\right)^{2}+r_{c}^{2}\right]} .
$$

Here $\varepsilon$ is the same as in (3.1). When $r \geqslant 1$ we replace the denominator $\exp \left[\left(x_{i j}+y_{i j}\right)^{2}+\right.$ $\left.r_{c}^{2}\right]$ with $\exp \left[x_{i j}^{2}+y_{i j}^{2}+r_{c}^{2}\right]$ for a fast decay of the solution. The formulation (3.5) in cylindrical coordinates is

$$
U_{\theta}(r, \theta)=\varepsilon h(r, \theta) \cos (\theta+3 \pi / 4), \quad U_{r}(r, \theta)=\varepsilon h(r, \theta) \sin (\theta+3 \pi / 4)
$$

where $h(r, \theta)=r(\cos (\theta)+\sin (\theta)) / \exp \left(r^{2}(\cos (\theta)+\sin (\theta))^{2}+r_{c}^{2}\right)$.

\subsection{Time evolution}

The time evolution of the vortex can be seen in Figure 4. The kinetic energy of the vortex spread evenly to the area surrounding it. At $\mathrm{t}=4000$ the vortex seems only slightly weaker. In an isolated vortex one has an inner vortex core $\left(r<r_{c}\right)$ which is rotating as a solid body. In the outer part $\left(r>r_{c}\right)$, both the strain rate (with non-zero eigenvalues) and the vorticity is present, see the first remark in section 2.2 . Thus, the vortex will be damped from outside leaving the vortex core undamped but with a shrinking core radius. This is exactly what is seen in Figure 4, and also consistent with the finding of Wallin \& Girimaji (2000) for turbulent decay of wing tip vortices.

The white noise computation is displayed in Figure 5. It decays quickly and has almost vanished at $t=1000$. A closer look at what remain of the white noise at $t=400$ and $\mathrm{t}=1000$ reveal several vorticity like flow structures. The time development of the special divergence free case is displayed in Figure 6 . The flow develops into two counter clockwise rotating vortices. These vortices remain for a long time and decay slowly.

Remark: The result of these three calculations is consistent with the theory in Section 2. The fact that even if one starts the calculation with a non-vortex structure (as in Figures 5 and 6), one ends up with only vortex like structures is particularly striking. This could be a scenario in which energy is transferred from non-vorticity modes to vorticity modes as mentioned earlier. 


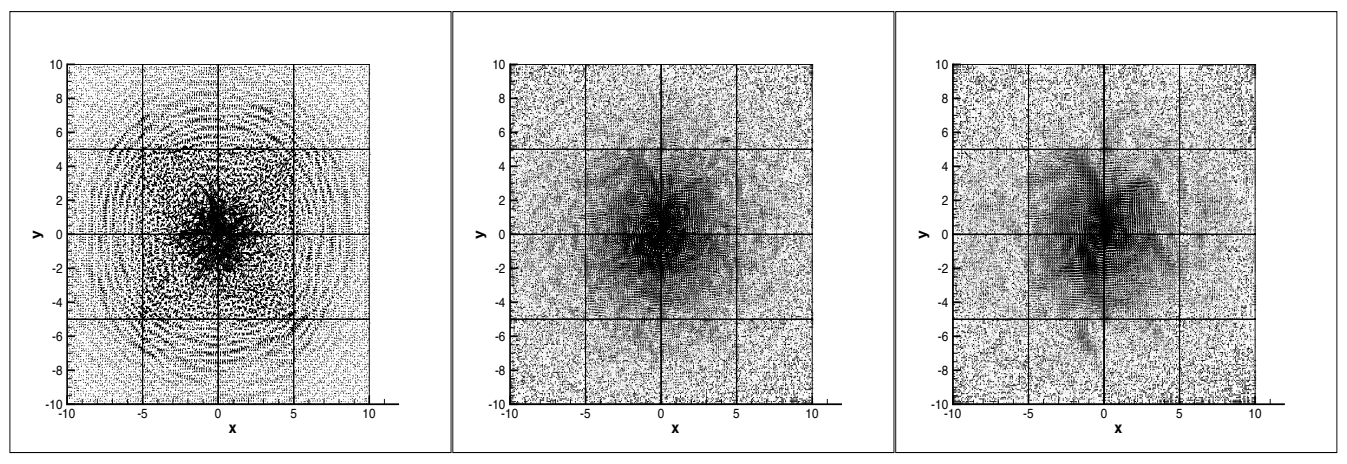

(a) $\mathrm{t}=8$

(b) $\mathrm{t}=400$

(c) $t=1000$

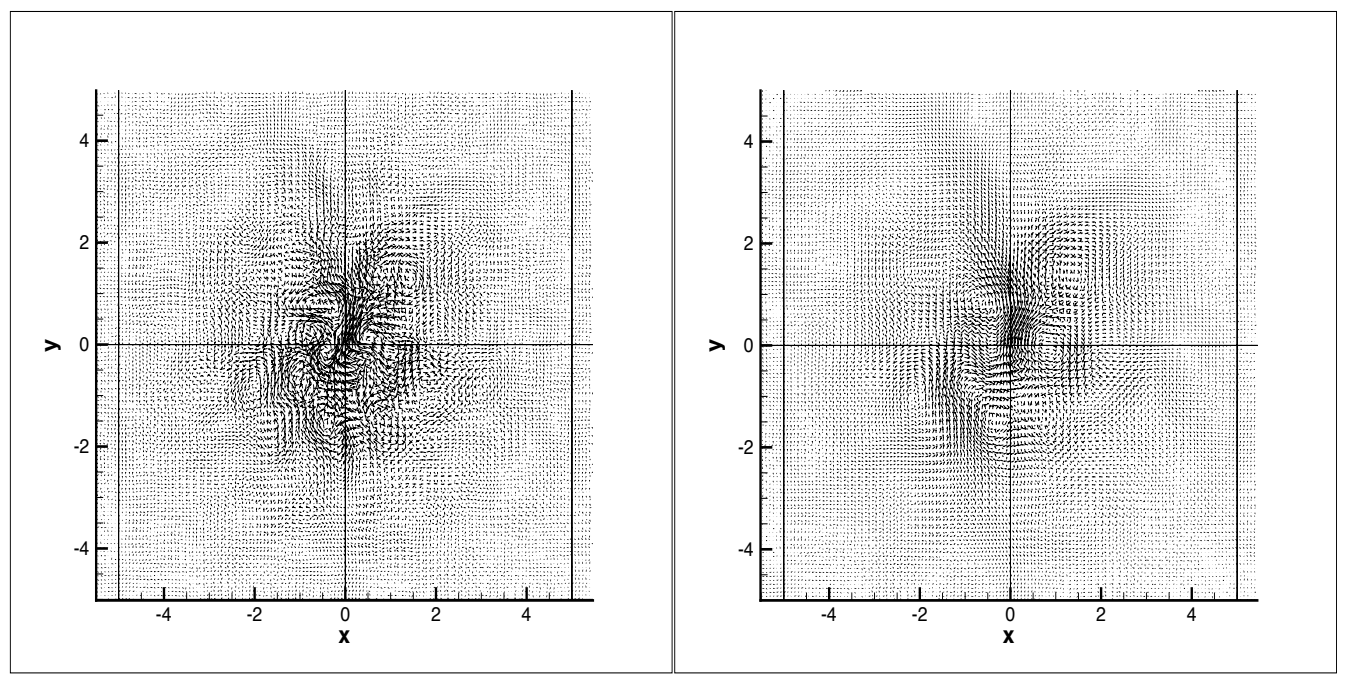

(d) $t=400$ zoomed

(e) $\mathrm{t}=1000$ zoomed

Figure 5: Time evolution of the white noise for $R e=1000$. The white noise decays quickly. At $t=400$ and $t=1000$ only vorticity like flow structures remain.

\subsection{Energy decay}

The kinetic energy of the global system $E=\rho \frac{u^{2}+v^{2}+w^{2}}{2}$ as a function of time, normalized by the initial kinetic energy, is shown in Figure 7. The energy decay of white noise is clearly faster than the energy decay of the wake vortex and the divergence free flow case. The fast decay is predicted by the theory since white noise has large gradients initially compared to the other initial conditions. Note that once the white noise has developed into a flow consisting mainly of vorticity, see Figure 5, the decay stops.

The energy of the vortex decrease slowly with time which agrees with the results obtained in Winckelmans et al. (2006). Despite the fact that the wake vortex flow with $\mathrm{Re}=100$ looks weaker at $\mathrm{t}=4000$ more than $90 \%$ of the kinetic energy in the global system remain.

The energy decay of the divergence free case is faster than the vortex energy decay but slower than the white noise energy decay. The decay is semi-fast initially when the velocity 


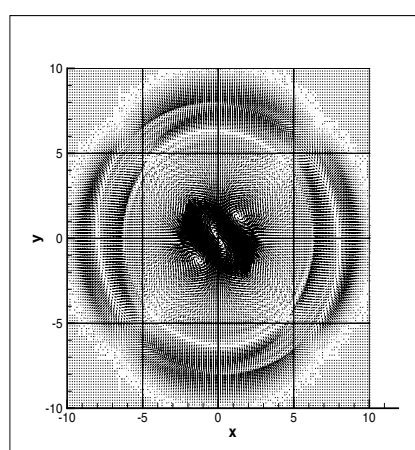

(a) $\mathrm{t}=7$

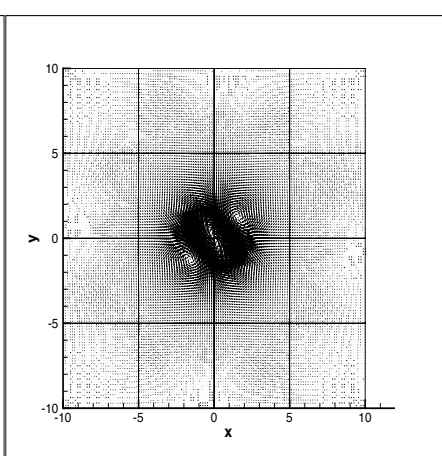

(b) $t=1000$

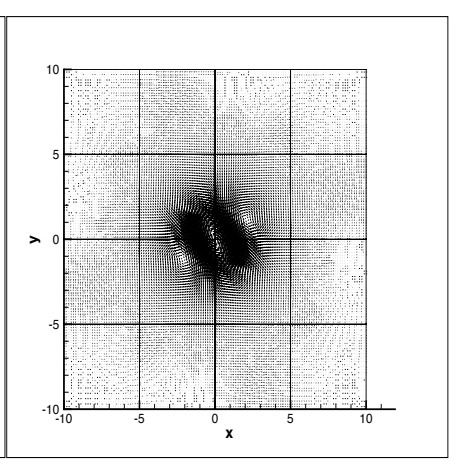

(c) $\mathrm{t}=4000$

Figure 6: Time evolution of the divergence free case for $R e=1000$. A wave leaves the domain at $\mathrm{t}=10$. The remaining counter clockwise rotating vortices decay slowly.

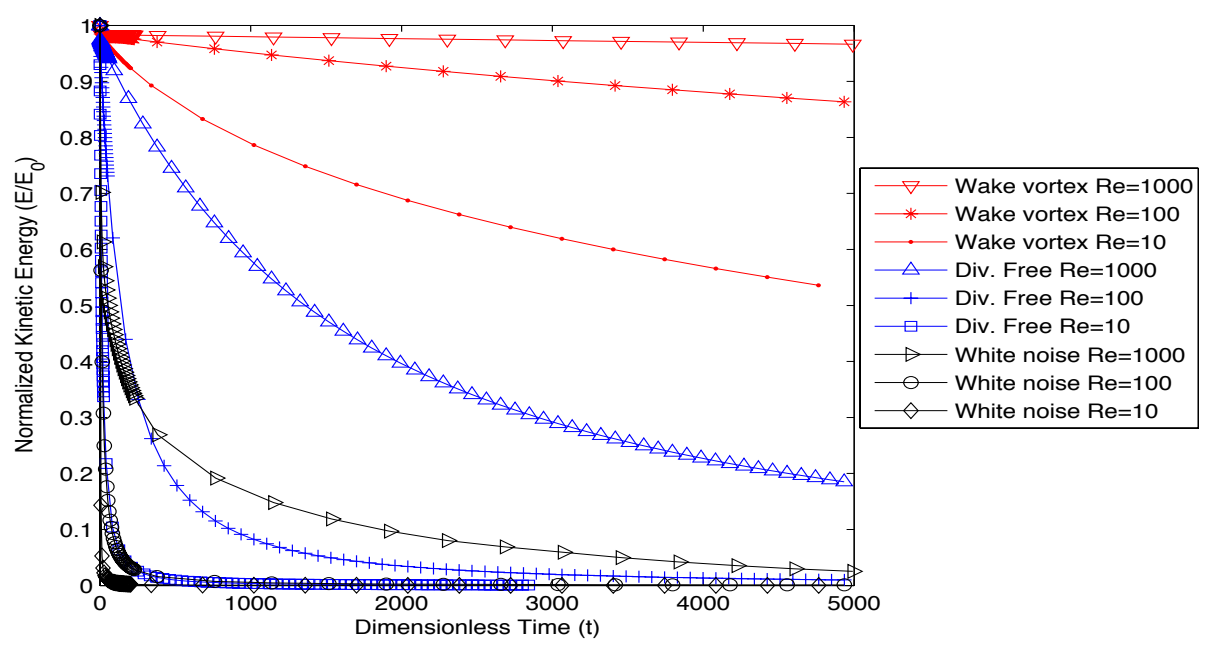

Figure 7: Normalized kinetic energy decay for the 2D vortex, divergence free and white noise cases as a function of time. The vortex has the least energy decay.

gradients are strong and decreases as the flow becomes dominated by the two counter rotating vortices. Once the vortices have formed, the decay slow down significantly.

For all three cases, computing with a lower/higher Reynolds number consistently resulted in more/less energy decay for all three cases.

Remark: The result of the calculations, i.e that vortex like structures decay slowly and other flow structures relatively fast is consistent with the theory in Section 2. The results are also consistent with the asymptotic decay rates obtained in Hoff \& Zumbrum (1995) for the Cauchy problem, if one takes into account the fact that we are computing on a finite domain. (On a finite domain, the acoustic part will radiate to infinity and decay rapidly). The results of Hoff \& Zumbrum (1995) also indicate that the transfer of energy between the vorticity modes and other modes is small, which again is consistent with our computational results. 


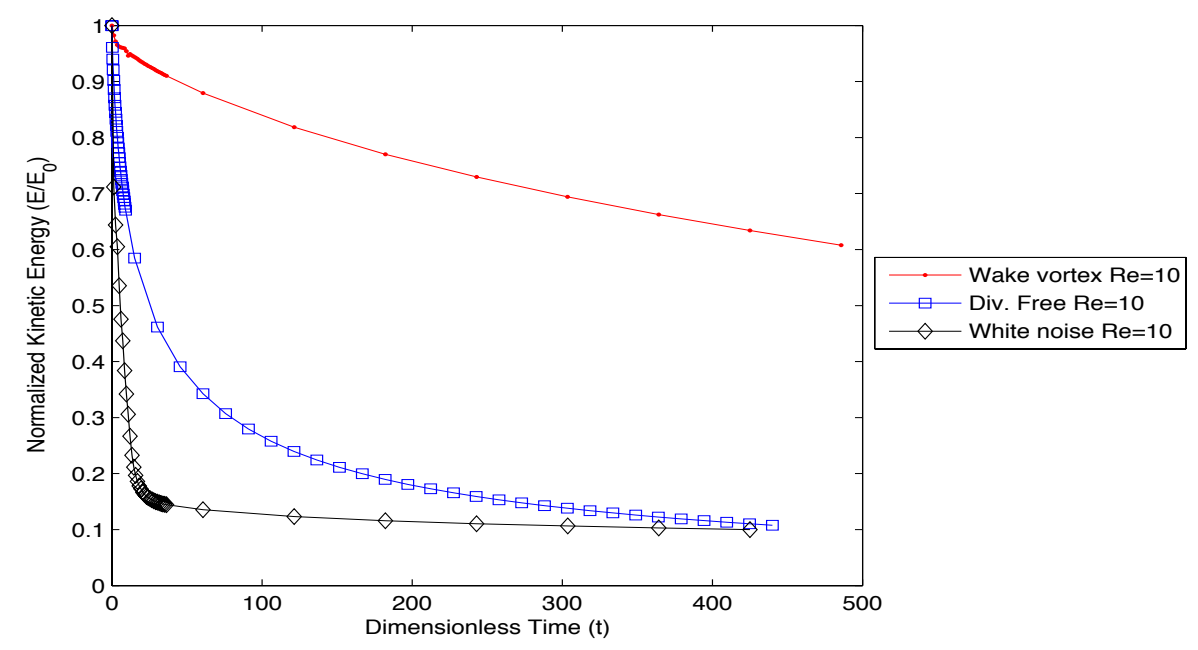

Figure 8: Normalized kinetic energy decay for the 3D vortex, divergence free and white noise cases as a function of time. The vortex has the least energy decay also in 3D.

\subsection{Validation of the results}

To confirm the results obtained in two dimensions, also three dimensional calculations were made. The initial velocities are defined as:

$$
\begin{gathered}
\text { Vortex }: u_{i j k}=\frac{-\varepsilon \cdot y_{i j k}}{f(x, y, z)}, v_{i j k}=\frac{\varepsilon \cdot x_{i j k}}{f(x, y, z)}, w_{i j k}=0 \\
\text { White noise }: u_{i j k}=\frac{\varepsilon \cdot \text { random }}{f(x, y, z)}, v_{i j k}=\frac{\varepsilon \cdot \text { random }}{f(x, y, z)}, w_{i j k}=0 \\
\text { Divergence free }: u_{i j}=\varepsilon \frac{x_{i j}+y_{i j}}{g(x, y, z)}, v_{i j}=-\varepsilon \frac{x_{i j}+y_{i j}}{g(x, y, z)}, w_{i j k}=0
\end{gathered}
$$

where $f(x, y, z)=x_{i j k}^{2}+y_{i j k}^{2}+z_{i j k}^{2}+r_{c}^{2}$ and $g(x, y, z)=\exp \left[\left(x_{i j}+y_{i j}+z_{i j}\right)^{2}+r_{c}^{2}\right]$. The same parameter values as in two dimensions were used. Note that the flow is truly three dimensional due to the $z$-dependence in $f(x, y, z)$ and $g(x, y, z)$ even though the velocity in the z-direction is initially zero. Note also that (3.9) is, strictly speaking, no longer divergence free due to the $z$ dependence.

The focus of the computations in three dimensions is to verify the relevance of the computations in two dimensions. Far field boundary conditions are used on all outer boundaries, except in the z-direction where periodic boundary conditions are used.

The energy of the global system as a function of time is shown in Figure 8. In all three computations the energy decay is similar to and hence validate the energy decay obtained from the two dimensional computations.

\section{Discussion}

The main result of the mathematical analysis, relation (2.13), describes how the disturbance energy (the deviation from the constant background field) decay (or do not decay). The right hand side of (2.13) is diagonalised such that each eigenvalue multiplies a quadratic form. Each quadratic form, shown in (2.14), consists of linear combinations 
of the gradients of the flow variables. The eigenvalues and quadratic forms can be organized in two groups. Group one has non-zero positive eigenvalues and group two has zero eigenvalues and correspond exactly to the vorticity modes. For easy of explanation we denote the modes in group one, the 'decay' modes.

We start by considering the energy decay only. Firstly, assume that all the quadratic forms/modes are non-zero. Due to the non-zero eigenvalues related to the 'decay' modes, we have energy decay. Secondly, assume that the 'decay' modes are zero, while the vorticity modes are non-zero. Then, since the eigenvalues related to the vorticity modes are zero, the energy will stay constant even though the corresponding quadratic forms/modes are non-zero. Note that if all the eigenvalues were non-zero, no possibility for a constant energy would exist.

Now we include energy transfer in the discussion. Consider the second case above which at a particular time do not yield energy decay, i.e. all the energy reside in the vorticity modes. If the energy from the the vorticity modes is transferred to the 'decay' modes, we have energy decay in the next instant. If the transfer is large, we will have a large decay, if the transfer is small we will have a small decay. The reversed scenario is also possible, i.e. that energy may transfer from 'decay' modes to vorticity modes which will decrease the energy decay from a high level to a low level.

The results discussed above have implications for the practical treatment (both the removal and the preservation) of energy content in flow structures. For a general flow structure from which one wants to remove energy, one could aim for transferring energy from the vorticity modes into the 'decay' modes, and use the natural decay mechanism discovered in this paper as guidance.

\section{Conclusions}

We have analysed the energy decay of small scale disturbances in laminar flow by using the energy-method. An equation for the energy decay rate in terms of gradients and a dissipation matrix was derived. Zero eigenvalues of the dissipation matrix multiplied the vorticity components.

The zero eigenvalues multiplying the vorticity components suggest that flows with most of the energy in the vorticity components decay slowly. It also mean that small scale disturbances will have most of their energy in vorticity form after a long time.

Numerical simulations verified that the energy decay is slow for vortices compared to the white noise and divergence free flow cases. The simulations also verified that the energy transfer from the vorticity components to other modes is slow, leading to a slow overall decay.

The existence of zero eigenvalues multiplying the vorticity component of the flow is probably one of the main reasons for the slow decay of energy in vortex like structures in fluid mechanics.

The energy transfer between vorticity modes and non-vorticity modes was investigated numerically. This part could possibly be improved by an even more advanced analysis and we hope that the finding in this paper will inspire others to do that.

\section{REFERENCES}

Abarbanel, S. \& Gottlieb, D. 1981 Optimal time splitting for two- and three-dimensional Navier-Stokes equations with mixed derivatives. Journal of Computational Physics 41 (1), 1-33.

Anderson, J. D. 1991 Fundamentals of Aerodynamics, 2nd edition. McGraw-Hill, Inc. 
Balmforth, NJ, Smith, SGL \& Young, WR 2001 Disturbing vortices. Journal of Fluid Mechanics 426, 95-133.

Beggs, P. J., Selkirk, P. M. \& Kingdom, D. L. 2004 Identification of von karman vortices in the surface winds of heard island. Boundary-Layer Meteorology 113 (2), 287-297.

Breitsamter, C. 2011 Wake vortex characteristics of transport aircraft. Progress in Aerospace Sciences 47 (2), 89-134.

Carpenter, M. H., Nordström, J. \& Gottlieb, D. 1999 A stable and conservative interface treatment of arbitrary spatial accuracy. Journal of Computational Physics 148 (2), 341365 .

Chen, K. K., Colonius, T. \& Taira, K. 2010 The leading-edge vortex and quasisteady vortex shedding on an accelerating plate. Physics of Fluids 22 (3), 1-11.

Davoudzadeh, F., McDonald, H. \& Thompson, B. E. 1995 Accuracy evaluation of unsteady cfd numerical schemes by vortex preservation. Computers and Fluids 24 (8), 883-895.

Erlebacher, G., Hussaini, M. Y. \& Shu, C. W. 1997 Interaction of a shock with a longitudinal vortex. Journal of Fluid Mechanics 337, 129-153.

Gerz, T \& Holzapfel, F 1999 Wing-tip vortices, turbulence, and the distribution of emissions. AIAA Journal 37 (10), 1270-1276.

Gerz, T, Holzapfel, F \& DarracQ, D 2002 Commercial aircraft wake vortices. Progress in Aerospace Sciences 38 (3), 181-208.

Greene, GC 1986 An Approximate Model for Vortex Decay in the Atmosphere. Journal of Aircraft 23 (7), 566-573.

Gustafsson, B., Kreiss, H. O. \& Oliger, J. 1995 Time Dependent Problems and Difference Methods. Academic Press.

Gustafsson, B \& Sundström, A 1978 Incompletely Parabolic Problems in Fluid-Dynamics. SIAM Journal on Applied Mathematics 35 (2), 343-357.

Gustavsson, LH 1991 Energy Growth of 3-Dimensional Disturbances in Plane Poiseuille Flow. Journal of Fluid Mechanics 224, 241-260.

Hagstrom, T. \& Lorenz, J. 1995 All-time existence of smooth solutions to PDEs of mixed type and the invariant subspace of uniform states. Advances in Applied Mathematics 16, $219-257$.

Hagstrom, T. \& Lorenz, J. 2002 On the stability of approximate solutions of hyperbolicparabolic systems and the all-time existence of smooth, slightly compressible flows. Indiana Univiversity Mathematics Journal 51, 1339-1387.

Hoff, D. \& Zumbrum, K. 1995 Multidimensional diffusion waves for the Navier-Stokes equations of compressible flow. Indiana Univiversity Mathematics Journal 44 (2), 603-676.

Holland, W. R. 1978 The role of mesoscale eddies in the general circulation of the ocean numerical experiments using a wind-driven quasi-geostrophic model. Journal of Physical Oceanography 8, 363-392.

Holzapfel, F, Hofbauer, T, Darracq, D, Moet, H, Garnier, F \& Gago, CF 2003 Analysis of wake vortex decay mechanisms in the atmosphere. Aerospace Science and Technology 7 (4), 263-275.

Kantha, L. 2010 Decay of Aircraft Wake Vortices Under Daytime Free Convective Conditions. Journal of Aircraft 47 (6), 2159-2164.

KIM, J-H. \& KIM, C. 2011 Computational Investigation of Three-Dimensional Unsteady Flowfield Characteristics Around Insects' Flapping Flight. AIAA Journal 49 (5), 953-968.

Koszalka, I., LaCasce, J. H., Andersson, M., Orvik, K. A. \& Mauritzen, C. 2011 Surface circulation in the Nordic Seas from clustered drifters. Deep-Sea Research Part IOceanographic Research Papers 58 (4), 468-485.

Kreiss, H. O. \& Lorenz, J. 1989 Initial Boundary Value Problems and the Navier-Stokes Equations. John Wiley \& Sons.

Kundu, P. \& Cohen, I. 2001 Fluid mechanics, 2nd edition. Elsevier Academic Press.

Liu, Y., Liu, N. \& Lu, X. 2009 Numerical Study of Two-Winged Insect Hovering Flight. Advances in Applied Mathematics and Mechanics 1 (4), 481-509.

Magnusson, M. \& Smedman, A. 1999 Air flow behind wind turbines. Journal of Wind Engineering and Industrial Aerodynamics 80 (1-2), 169-189.

Matthaeus, Wh, Stribling, WT, Martinez, D, Oughton, S \& Montgomery, D 1991 
Decaying, 2-Dimensional, Navier-Stokes Turbulence at Very Long Times. Physica D 51 (13), 531-538.

Mattsson, K, Svard, M \& Nordstrom, J 2004 Stable and accurate artificial dissipation. Journal of Scientific Computing 21 (1), 57-79.

McCormack, P. D. \& Crane, L. 1973 Physical Fluid Dynamics. Academic press.

Nordström, J 1995 The Use of Characteristic Boundary-Conditions for the Navier-Stokes Equations. Computers $\&$ Fluids 24 (5), 609-623.

Nordström, J. \& CARPenter, M.H. 1999 Boundary and interface conditions for high-order finite-difference methods applied to the Euler and Navier-Stokes equations. Journal of Computational Physics 148, 621-645.

Nordström, J. \& CARpenter, M. H. 2001 High-order finite difference methods, multidimensional linear problems, and curvilinear coordinates. Journal of Computational Physics 173 (1), 149-174.

Nordström, J., Gong, J., van Der Weide, E. \& Svärd, M. 2009 A stable and conservative high order multi-block method for the compressible Navier-Stokes equations. Journal of Computational Physics 228 (24), 9020-9035.

Nordström, J. \& SvÄRD, M. 2005 Well-posed boundary conditions for the navier-stokes equations. SIAM Journal on Numerical Analysis 43, 1231-1255.

Ponta, F. L. 2010 Vortex decay in the Karman eddy street. Physics of Fluids 22 (9).

Proctor, F. H. 1998 The NASA-Langley wake vortex modelling effort in support of an operational aircraft spacing system, AIAA paper 98-0589. In 36th AIAA Aerospace Sciences Meeting and Exhibit.

Pullin, DI \& Saffman, PG 1998 Vortex dynamics in turbulence. Annual Review of Fluid Mechanics 30, 31-51.

Rossow, VJ 1999 Lift-generated vortex wakes of subsonic transport aircraft. Progress in Aerospace Sciences 35 (6), 507-660.

SARPKAYA, T 1998 Decay of wake vortices of large aircraft. AIAA Journal 36 (9), 1671-1679.

SPAlart, PR 1998 Airplane trailing vortices. Annual Review of Fluid Mechanics 30, 107-138.

Svärd, M., Carpenter, M. H. \& Nordström, J. 2007 A stable high-order finite difference scheme for the compressible Navier-Stokes equations, far-field boundary conditions. Journal of Computational Physics 225 (1), 1020-1038.

SvÄrd, M. \& NordströM, J. 2008 A stable high-order finite difference scheme for the compressible Navier-Stokes equations. No-slip wall boundary conditions. Journal of Computational Physics 227 (10), 4805-4824.

Wallin, S. \& GiRimaji, SS. 2000 Evolution of an isolated turbulent trailing vortex. AIAA Journal 38 (4), 657-665.

White, F. M. 1974 Viscous Fluid Flow. McGraw-Hill, Inc.

Winckelmans, G., Cocle, R., Dufresne, L., Capart, R., Bricteux, L., Daeninck, G., Lonfils, T., Duponcheel, M., Desenfas, O. \& Georges, L. 2006 Direct numerical simulation and large-eddy simulation of wake vortices: Going from laboratory conditions to flight conditions. In In European Conference on Computational Fluid Dynamics, ECCOMAS CFD.

Winter, B. \& Bourqui, M. S. 2011 The impact of surface temperature variability on the climate change response in the Northern Hemisphere polar vortex. Geophysical Research Letters 38.

Wu, T. Y. 2011 Fish Swimming and Bird/Insect Flight. In Annual Review of Fluid Mechanics, Annual Review of Fluid Mechanics, vol. 43, pp. 25-58.

YEN, S-C. 2011 Aerodynamic Performance and Shedding Characteristics on a Swept-back Wing. Journal of Marine Science and Technology-TAIWAN 19 (2), 162-167.

Zhang, Y., Pedlosky, J. \& Flierl, G. R. 2011 Shelf Circulation and Cross-Shelf Transport out of a Bay Driven by Eddies from an Open-Ocean Current. Part I: Interaction between a Barotropic Vortex and a Steplike Topography. Journal of Physical Oceanography 41 (5), 889-910.

Zhou, J, Adrian, RJ, Balachandar, S \& Kendall, TM 1999 Mechanisms for generating coherent packets of hairpin vortices in channel flow. Journal of Fluid Mechanics 387, 353396. 